\title{
El Smartphone en el campo escolar: análisis del discurso científico producido en los artículos que reposan en la base de datos de la Red de Revistas Científicas de América Latina y el Caribe, España y Portugal (REDALYC)
}

The Smartphone in the school field: analysis of the scientific discourse produced in the articles of the Red de Revistas Científicas de América Latina y el Caribe, España y Portugal (REDALYC)

Smartphone no campo escolar: análise do discurso científico produzido nos artigos disponíveisna base de dados da Red de Revistas Científicas de América Latina y el Caribe, España y Portugal (REDALYC)

Rafael Alberto González González

Doutorando da Universidade Federal da Bahia, Salvador, Bahia, Brasil. cinco.venezuela@gmail.com - https://orcid.org/0000-0003-1544-925X

María Helena Silveira Bonilla

Professora Doutora da Universidade Federal da Bahia, Salvador, Bahia, Brasil. bonillabr@gmail.com - http://orcid.org/0000-0002-0137-6363

Recebido em 18 de agosto de 2020

Aprovado em 10 de dezembro de 2020

Publicado em 05 de maio de 2021

\section{RESUMEN}

La escuela del siglo XXI atraviesa un singular proceso de mutación; la entrada en escena de lo digital (en términos genéricos) y del smartphone (en términos particulares), ha posibilitado la emergencia de una serie de nóveles realidades cuyos principales trazos se han venido constituyendo, en los más recientes lustros, en el campo escolar. A tales efectos, el discurso científico ha venido produciendo, en la intersección temática smartphone-escuela, unas regularidades enunciativas, un topoi, que ha pasado a definir las fronteras de lo enunciable/pensable en el marco de ese particular campo de saber que fusiona lo tecnológico con lo escolar. En tal sentido, el corriente trabajo se detiene a examinar, a través de las posibilidades de análisis que ofrece el Análisis Crítico del Discurso (ACD), 28 artículos que forman parte de la base de datos de la Red de Revistas Científicas de América Latina y el Caribe, España y Portugal (REDALYC). Al caso, se identificaron las regularidades discursivas que tipifican la intersección temática objeto de interés (smartphone-escuela), perfilando, de manera preliminar, el campo enunciativo instrumental-utilitario a partir del cual se 


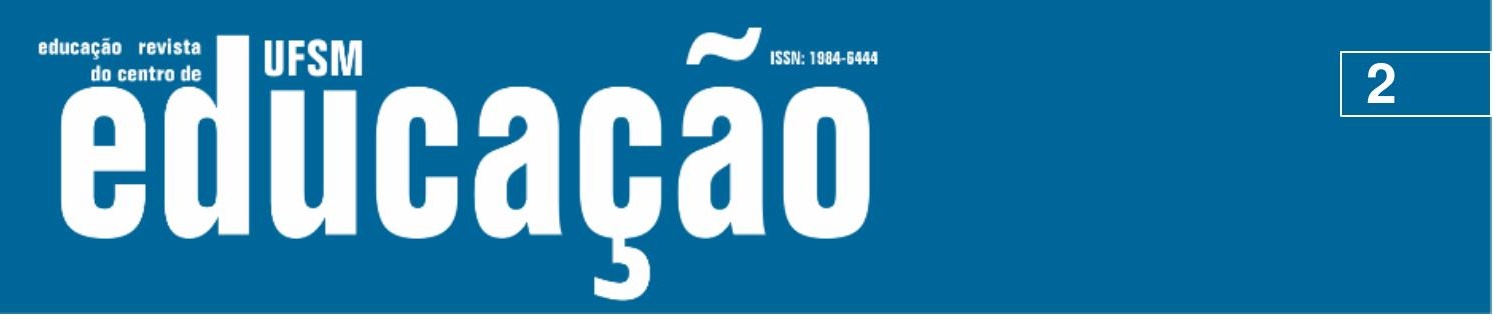

ISSN: 1984-6444 | http://dx.doi.org/10.5902/1984644453236

constituyen las relaciones de saber-poder a través de las cuales lo científico-escolar viene pensando, enunciando, el eje temático en consideración.

Palavras-chave: Smartphone; Escuela; Discurso Científico; Poder-Saber.

\section{ABSTRACT}

Twenty-first century school is having a unique transformation; The staging of the technological (in generic terms) and smartphones (in particular terms), triggered the emergence of new realities whose main features were constituted, in the last decades, in the scholar field. In this matter, scientific discourse has been producing some enunciative regularities in smartphone-school thematic intersection, a topoi, which has come to define the boundaries of the enunciable / thinkable in the framework of that particular field of knowledge that merges the technological with the academics. In that sense, this work examine, through the Critical Discourse Analysis (CDA), 28 articles that are part of the database of the Red de Revistas Científicas de América Latina y el Caribe (REDALYC). The discursive regularities that typify the thematic intersection smartphone-school (object of interest) were identified, outlining, in a preliminary way, the enunciative field instrumental-utilitarian from which knowledge-power relations are constituted through which the scientific-scholar has been thinking, stating, the thematic axis under consideration.

Keywords: Smartphone; School; Scientific Discourse; Knowledge-Power.

\section{RESUMO}

A escola do século 21 está passando por um processo de mutação único; $A$ entrada em cena do digital (em termos genéricos) e do smartphone (em termos particulares), permitiu o surgimento de uma série de novas realidades cujas principais características se constituíram, nas últimas décadas, no campo escolar. Para tanto, o discurso científico vem produzindo algumas regularidades enunciativas, na intersecção temática do smartphone-escola, um topoi que passou a definir os limites do enunciável/pensável no marco daquele determinado campo de conhecimento que mescla o tecnológico com a escola. Nesse sentido, o presente trabalho examina através da Análise Crítica do Discurso (ACD), 28 artigos que fazem parte do banco de dados da Red de Revistas Científicas da América Latina e Caribe, España y Portugal (REDALYC). Foram identificadas as regularidades discursivas que tipificam a intersecção temática smartphone-escola (objeto de interesse), delineando de forma preliminar o campo enunciativo instrumental-utilitário a partir do qual se constituem as relações de saber-poder através das quais o científico-escolar vem pensando, enunciando, o eixo temático em consideração.

Palavras-chave: Smartphone; Escola; Discurso Científico; Saber-Poder. 


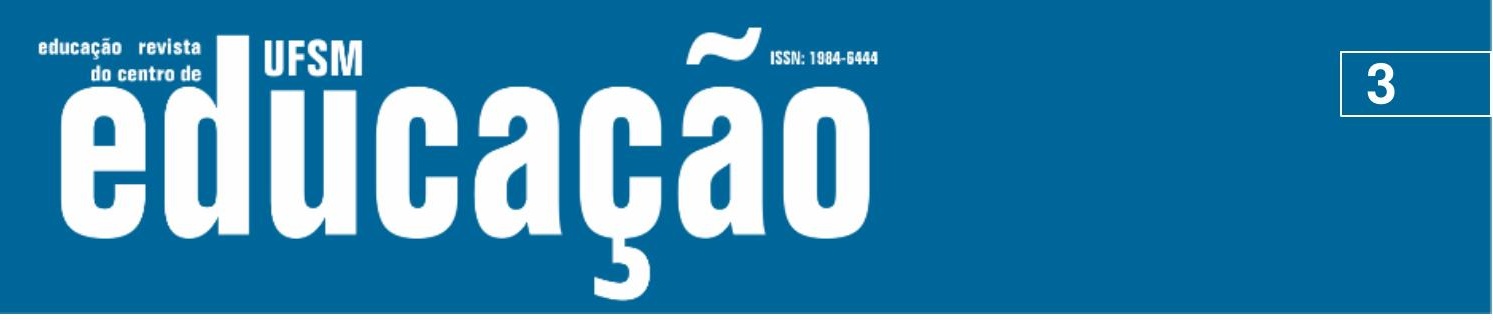

ISSN: 1984-6444 | http://dx.doi.org/10.5902/1984644453236

\section{Introducción}

La aldea global viene transformándose de manera vertiginosa; la entrada en escena de la revolución digital, a partir de las últimas décadas del siglo $\mathrm{XX}$, ha venido a confirmar el fraguado de un nuevo epocal en la revolucionaria marcha de los tiempos modernos. En tales términos, el larvado de la denominada sociedad-red (CASTELLS, 2006) se erige como el fenómeno socio-histórico más trascendental en el tránsito de la modernidad temprana (industrial) a la modernidad tardía (informacional), lo cual ha venido a configurar una nueva experiencia-planetaria en la cual lo digital se afirma como el epicentro de las múltiples mudanzas que, en casi todas las aristas del quehacer social, se patentizan contemporáneamente.

En tales términos, un tópos en el cual logran constatarse, de manera clara, los avatares que trae consigo el mundo digital (NEGROPONTE, 1995), resulta aquel que tiene a la escuela (de manera particular) y la educación (de manera general), como eje-central de la formación y la transmisión socio-cultural de las sociedades modernas, toda vez que basta pasearse por la abundante producción científica que existe en términos de la relación tecnologías digitales-escuela, para constatar el denso trabajo investigativo que se viene realizando a los fines de dimensionar la metamorfosis operada, en relación a lo escolar, en la transición del siglo XX al XXI. Al respecto, la escuela de la sociedad-red no solamente se encuentra en un proceso de reconfiguración que permea cada una de sus fibras constitutivas (el currículo, la didáctica, la formación de profesores, la producción de contenidos, el rol docente, etc.), sino que incluso la propia existencia de la institución, a la luz de la función social que le resulta correlativa (educar), atraviesa tiempos complejos, que invitan a repensarla, en virtud de las posibilidades educativo-tecnológicas que han ido germinando a partir de la inteligencia artificial, la robótica humanoide y/o la virtualidad.

A la luz de lo apuntado, destaca la importancia analítica que han adquirido las tecnologías móviles, en específico el smartphone, en los procesos de aprendizaje, socialización, gestión, innovación, etc., que acontecen o se relacionan transversalmente con la escuela, toda vez que el nivel de penetración poblacional 


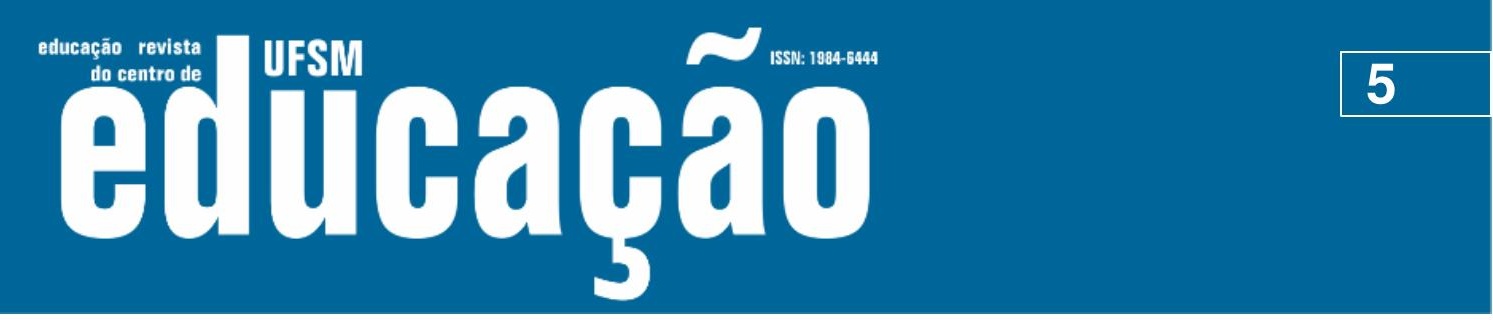

ISSN: 1984-6444 | http://dx.doi.org/10.5902/1984644453236

\section{Metodología}

La corriente pesquisa se encuadra en una modalidad de investigación de tipo documental. A tales efectos, los resultados obtenidos se desprenden del análisis semántico de un corpus textual de 28 artículos científicos que, en lo atinente al smartphone, reposan en la base de datos (repositorio digital) de la Red de Revistas Científicas de América Latina y el Caribe, España y Portugal (REDALYC). En tal sentido, los documentos objeto de abordaje fueron seleccionados/examinados a partir de un doble proceso de compilación/análisis que, de manera sistemática, se ajustó a las dos siguientes fases, a saber:

-Primera fase: Consistió en la búsqueda y selección del material documental objeto de interés. En tal sentido, se procedió a identificar, en la base de datos de REDALYC, los artículos científicos cuyo contenido coincidió con la palabra clave smartphone. Luego de ello, se aplicaron los filtros de búsqueda que ofrece el repositorio en cuestión, definiendo para ello tanto un criterio de selección de tipo disciplinar (artículos desarrollados en el campo escolar), como uno temporal (artículos producidos en el periodo 2015-2019), obteniendo un total de 274 artículos. Llegados a este punto, se examinó el título, resumen, palabras claves y resultados de cada uno de los documentos previamente identificados; a tales fines, se seleccionaron los artículos científicos que de manera neurálgica, central, se enfocaban en el estudio/comprensión del smartphone en el campo escolar. De tal manera, se obtuvieron los 28 artículos científicos que componen el corpus documental de la presente investigación.

-Segunda fase: Consistió en el procesamiento cuali-cuantitativo del contenido examinado en el referido corpus-documental. Para ello, se emprendió un proceso de categorización (CUENCA; HILFERTY, 2007) y la construcción de una red semántica (GARÓFALO; GALAGOVSKY, 2015), empleando para tales propósitos las posibilidades de procesamiento y tabulación documental que ofrece el software Atlas.ti, así como los preceptos metodológicos incardinados al Análisis Crítico del Discurso (VAN DIJK, 1994). Los insumos obtenidos a través de tales procedimientos objetivan el contenido temático, las 


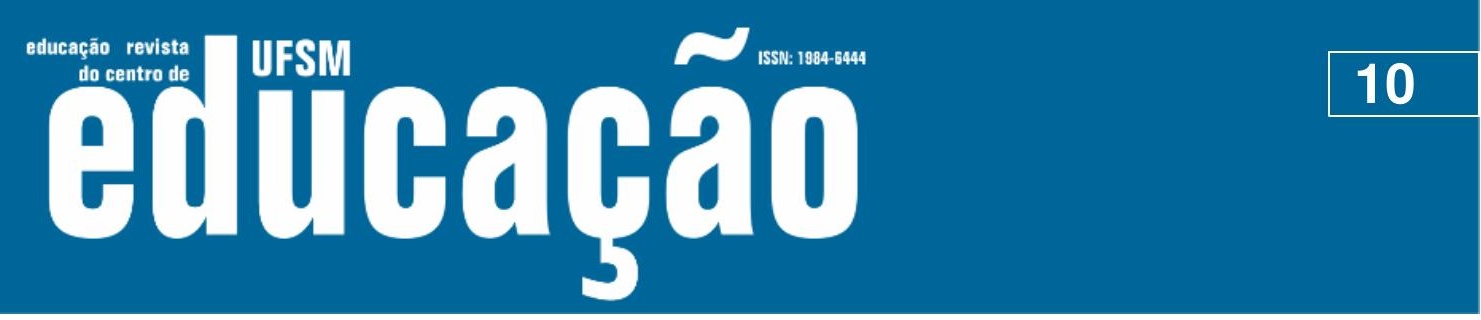

ISSN: 1984-6444 | http://dx.doi.org/10.5902/1984644453236

apreciar los múltiples puntos de conexión que existen, en términos de poder, entre el discurso científico y lo escolar. He allí delimitada una importante intersección políticoepistémica que urge escudriñar/auscultar, en términos de lo que Basil Bernstein (1996) denominó la estructuración del discurso pedagógico, a los fines de rastrear las relaciones de fuerza, de poder, gracias a las cuales se configura el marco social de lo enunciable/pensable.

Ante lo apuntado, la escuela y el discurso científico emergen como dos de las principales instituciones que garantizan la (re)producción del orden del discurso, la (re)producción del control simbólico (BERNSTEIN, 1996), motivo por el cual los referidos dispositivos demandan ser estudiados/auscultados (estratégicamente), a los fines de identificar las relaciones de saber-poder, los intereses, que se producen/circulan socialmente a través (y procurando el control) del discurso. Así pues, la dupla en cuestión se consolida como una ineludible vitrina para visualizar el carácter agónico, las líneas de fuerza, que resultan inherentes a la emergencia/consolidación de una episteme, por lo cual su estudio comporta cardinal para la comprensión de las diferentes fronteras enunciativas que tornan posible que el científico-el docente (en tanto sujetos de verdad), lo escolar-lo científico (en tanto campos de saber) y la escuela-la ciencia (en tanto instancias instituyentes), participen de esa economía de lo decible que permea a la sociedad y gracias a la cual se objetiva un orden discursivo (y no otro). De allí que el estudio/abordaje de la relación discursopoder-escuela resulte capital para inteligir aquello que, en términos de la relación smartphone-escuela, se ha optado por examinar en la presente oportunidad.

\section{El smartphone en el campo escolar: regularidades discursivas}

El smartphone, ese singular dispositivo tecnológico que ha pasado a ocupar un papel cardinal en la cotidianidad de las sociedades contemporáneas, forma parte de una multiplicidad de procesos, de una colosal cantidad de fenómenos educacionales, cuya complejidad viene transformando, profundamente, la morfología de las sociedades líquidas. Basta examinar, al caso, el tipo de sinergia que favorece el smartphone (de un modo particular) y las tecnologías digitales móviles (de manera 


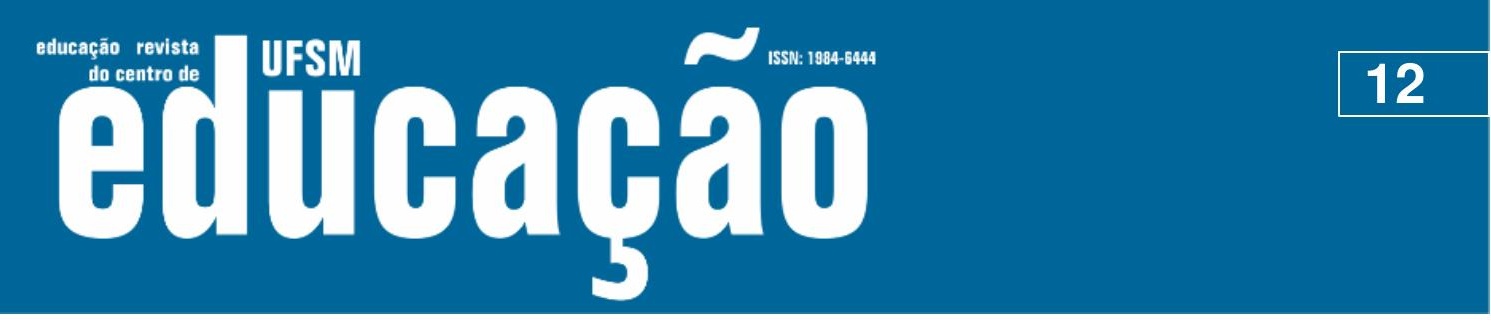

ISSN: 1984-6444 | http://dx.doi.org/10.5902/1984644453236

Tabla 01 - Penetración social del smartphone (enunciados)

\begin{tabular}{|c|c|c|c|}
\hline Autor & Enunciado & $\begin{array}{c}\text { Año, } \\
\text { Página }\end{array}$ & Observación \\
\hline $\begin{array}{l}\text { DE OLIVEIRA, } \\
\text { Rafael; } \\
\text { CARVALHO, } \\
\text { Mercedes }\end{array}$ & $\begin{array}{l}\text { No se necesitan investigaciones científicas para saber } \\
\text { que la sociedad actual se encuentra con el mundo de la } \\
\text { información en las manos, vía smartphone. }\end{array}$ & p.50 & $\begin{array}{l}\text { Traducciónnue } \\
\text { stra. }\end{array}$ \\
\hline $\begin{array}{l}\text { GOMES, Vânia; } \\
\text { SOARES, Antônio }\end{array}$ & $\begin{array}{l}\text { En efecto, el teléfono celular puede ser identificado como } \\
\text { un gadget que se torna cada vez más imprescindible no } \\
\text { solo para adolescentes, sino también para los adultos y } \\
\text { hasta los mismos niños. }\end{array}$ & $\begin{array}{l}2018 \\
p .421\end{array}$ & $\begin{array}{l}\text { Traducción } \\
\text { nuestra. }\end{array}$ \\
\hline $\begin{array}{l}\text { HÉRNANDEZ, } \\
\text { Nayesia }\end{array}$ & $\begin{array}{l}\text { La penetración estimada del teléfono móvil es del } 60 \% \text { a } \\
\text { escala mundial. }\end{array}$ & $\begin{array}{l}2017 \\
\text { p. } 150\end{array}$ & \\
\hline $\begin{array}{l}\text { DOMÍNGUEZ, } \\
\text { Claudia; } \\
\text { ORGANISTA, } \\
\text { Javier; LÓPEZ, } \\
\text { Maricela }\end{array}$ & $\begin{array}{l}\text { En el caso de los teléfonos inteligentes, también } \\
\text { conocidos como smartphones, el número de usuarios en } \\
\text { el sector estudiantil se ha incrementado en México. }\end{array}$ & $\begin{array}{l}2018 \\
\text { p.81 }\end{array}$ & \\
\hline $\begin{array}{l}\text { LÓPEZ, Juan } \\
\text { Carlos; CORREA, } \\
\text { Marisol }\end{array}$ & $\begin{array}{l}\text { (...) todos los participantes disponen de teléfono móvil, así } \\
\text { como conexión a internet desde casa y desde el móvil. }\end{array}$ & $\begin{array}{l}2019 \\
\text { p.12 }\end{array}$ & \\
\hline \begin{tabular}{|l|} 
RAMÍREZ- \\
MONTOYA, \\
María; GARCÍA- \\
PEÑALVO \\
\end{tabular} & $\begin{array}{l}\text { Múltiples informes y estudios avalan la creciente } \\
\text { importancia y penetración de los dispositivos móviles en } \\
\text { la sociedad, con especial atención a los smartphones. }\end{array}$ & 2017, & \\
\hline $\begin{array}{l}\text { YVON, Thomas; } \\
\text { LACERDA, } \\
\text { Gilberto }\end{array}$ & $\begin{array}{l}\text { Hay, de hecho, señales de democratización de los } \\
\text { smartphones. }\end{array}$ & $\begin{array}{l}2016 \\
\text { p.311 }\end{array}$ & $\begin{array}{l}\text { Traducciónnue } \\
\text { stra }\end{array}$ \\
\hline \begin{tabular}{|l|} 
GUTIÉRREZ, \\
María; SANTANA, \\
Josefina; PÉREZ, \\
Martha
\end{tabular} & $\begin{array}{l}\text { Según el Instituto Nacional de Estadísticas y Geografía } \\
\text { (Inegi), al menos } 9 \text { de cada } 10 \text { hogares tienen un teléfono } \\
\text { celular. }\end{array}$ & p.51 & \\
\hline MARTÍNEZ, José & $\begin{array}{l}\text { (...) los teléfonos celulares inteligentes }(\mathrm{TCl}) \text {, se hacen } \\
\text { más comunes en manos de estudiantes de secundaria y } \\
\text { universitarios, como lo reporta la Comisión Nacional de } \\
\text { Telecomunicaciones de Venezuela (CONATEL). }\end{array}$ & 2015 & \\
\hline $\begin{array}{l}\text { PEREIRA, } \\
\text { Luciana de } \\
\text { Cassia; } \\
\text { GOULART, Elias } \\
\end{array}$ & $\begin{array}{l}\text { La miniaturización de los dispositivos móviles de } \\
\text { comunicación, como es el caso del smartphone, agregada } \\
\text { la movilidad y la conectividad, están ganando espacios y } \\
\text { remodelando el hacer cotidiano de las personas. }\end{array}$ & 2019 & $\begin{array}{l}\text { Traducciónnue } \\
\text { stra }\end{array}$ \\
\hline \begin{tabular}{|l|} 
BRAZUELO, \\
Francisco; \\
CACHEIRO, \\
María \\
\end{tabular} & $\begin{array}{l}\text { Se estima que en } 2015 \text { la cifra global de usuarios de } \\
\text { internet ha alcanzado los } 3,2 \text { billones y más de } 7 \text { billones } \\
\text { de teléfonos móviles. }\end{array}$ & $\begin{array}{l}2015 \\
\text { p.02 }\end{array}$ & \\
\hline $\begin{array}{l}\text { MERINO, } \\
\text { Edgardo; } \\
\text { CABELLO, Jorge; } \\
\text { MERINO, Edgar }\end{array}$ & $\begin{array}{l}\text { El Instituto Nacional de la Juventud de Chile realizó el año } \\
2015 \text { un sondeo sobre Nuevas Tecnologías e Internet, de } \\
\text { él se desprende que los aparatos tecnológicos que más } \\
\text { mencionan tener las personas jóvenes son el teléfono } \\
\text { móvil }(97 \%) \text {. }\end{array}$ & & \\
\hline
\end{tabular}

Fuente: Elaboado por los autores 


\section{us: Autoabat

ISSN: 1984-6444 | http://dx.doi.org/10.5902/1984644453236

A partir de lo apuntado, queda claro el íntimo vínculo que se teje entre la importancia/notoriedad que ha adquirido el debate, la reflexión en lo atinente a la relación smartphone-escuela, y la correspondiente penetración poblacional que tipifica a la aludida tecnología móvil en el tejido social, en las dinámicas cotidianas, encargadas de configurar el quehacer contemporáneo. Al respecto, bien se puede asentir que el topoi a partir del cual se enuncia el nodo objeto de interés (smartphoneescuela), se construye tanto a partir de una perspectiva analítica que procura inteligir al predicho dispositivo en el marco de un tiempo histórico definido por lo digital (por la importancia adquirida por las TIC en el ensamblado de la nueva condición epocal), como por el correlativo/derivado factum inherente a la penetración demográfica alcanzada por dicho dispositivo en el tránsito de los más recientes lustros.

Ahora bien, destacados cada uno de los dos aspectos que definen el contexto en el cual se inscribe/enuncia la relación objeto de atención, se torna conveniente traer a colación las regularidades discursivas que aparecen con mayor fuerza, con insistencia, al momento de analizar la documentación compilada, toda vez que ello permite perfilar (más allá del par de ideas que se han desarrollado de manera preliminar) las singularidades a partir de las cuales se enuncia el smartphone en el campo escolar, identificando las unidades léxicas, las unidades de sentido, en virtud de las cuales se construye, se piensa, se viene reflexionando tan estratégico campo de saber.

A la luz de lo señalado, sirva referir los dos cuadros que presentamos a continuación a los fines de puntualizar la frecuencia estadística (absoluta), correspondiente tanto a la nube de palabras, a las unidades léxicas, que se repitieron con mayor intensidad en los artículos compilados (Cuadro 1), como a los códigos, las unidades de significación, que emergieron de la respectiva revisión documental (Cuadro 1). 


\section{$\sim$

ISSN: 1984-6444 | http://dx.doi.org/10.5902/1984644453236

Cuadro 3 - Red semántica

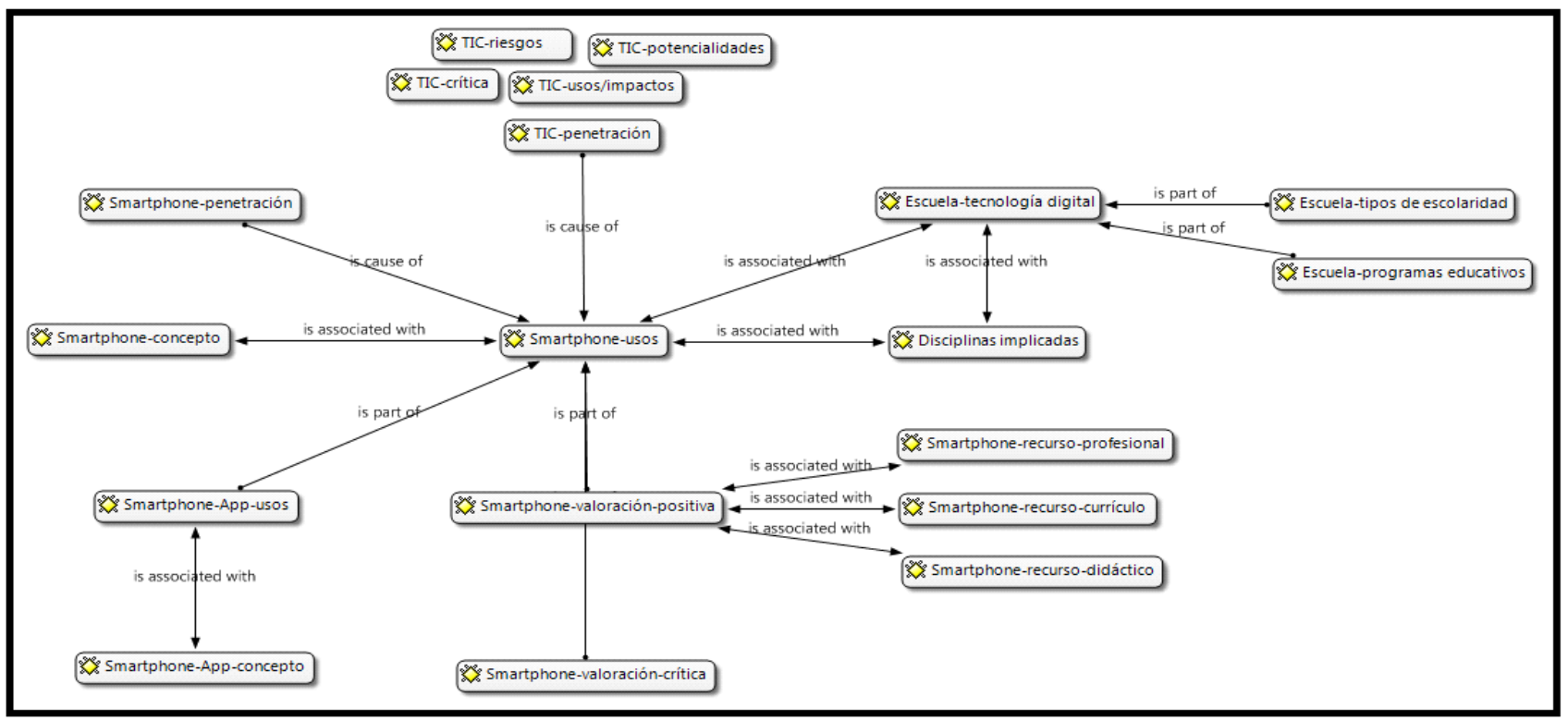

Fuente: Elaborado por los autores con el software Atlas.ti

En tal sentido, bien se puede asentir que el referente usos ocupa un papel capital en la red de significaciones que se edifican, discursivamente, en términos de la relación smartphone-escuela, tejiéndose alrededor de tal unidad de significación, en términos jerárquicos, tantos las valoraciones como los tópicos que componen la red semántica a través de la cual se enuncia, concibe, reflexiona, la relación smartphone-escuela. De lo señalado vale destacar, entonces, tanto la importancia léxica desempeñada por la familia de palabras que en lo atinente a los usos tipifica la enunciación del smartphone en el campo escolar, como el respectivo papel nodal que ocupa dicho referente en el marco de la producción de sentidos (las críticas/las positividades), a partir de las cuales se construye el discurso científico correspondiente al aludido campo de saber.

De igual modo, resulta de interés profundizar en la información atinente al referente usos e indicar que el mismo aparece asociado, en el corpus analizado, a cinco grandes vértices o campos de enunciación. Al respecto, el primer vértice hace referencia, como resulta esperable en un trabajo tematizado en lo escolar, a la dimensión pedagógico-didáctica que priva en el uso del smartphone, subrayando tanto 


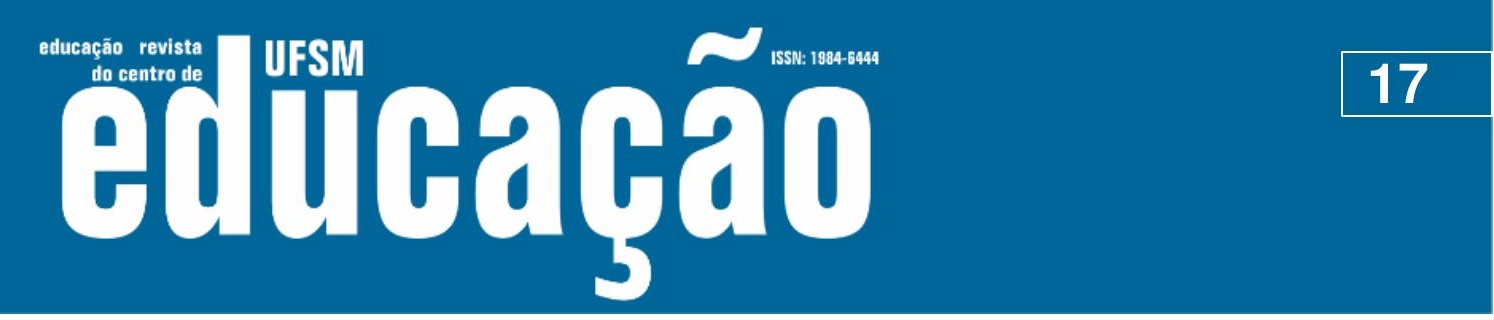

ISSN: 1984-6444 | http://dx.doi.org/10.5902/1984644453236

la utilidad de tal herramienta tecnológica en los procesos de enseñanza-aprendizaje (TANGARIFE, 2018; RODRÍGUEZ, 2017; PALAZÓN, 2015; YVON, 2016) y evaluación (SALCINES, 2015), que se desenvuelven a nivel escolar, como el correlativo valor pedagógico (PEREIRA, GOULART, 2019; MANCINAS, 2018; VÁSQUEZ, 2015; BRAZUELO, 2015; MERINO, 2017) que connota al referido dispositivo. De igual manera, la dimensión en consideración hace referencia a los usos particulares, típicos, que los sujetos escolares destinan al smartphone en términos de acceso a las redes sociales (GOMES, 2018; MEJÍA, 2017; GUTIÉRREZ, 2017; MERINO, 2017), uso de aplicativos o funciones para el registro, la consulta y/o la producción de información (DE OLIVEIRA, 2017; DEL CERRO, 2017; RAMÍREZ, 2017), la experimentación (DI LACCIO, 2017; MARTÍN-RAMOS, 2017; MARTÍNEZ, 2015), y/o el trabajo escolar con realidad virtual (GOMES, 2018) y realidad aumentada (RAMÍREZ, 2017).

El segundo vértice alude a las diversas modalidades o experiencias de escolaridad que se están desarrollando, en la actualidad, a través del aprendizaje móvil, el m-learning (RODRÍGUEZ, 2017; YVON, 2016; GUTIÉRREZ, 2017) y/o el aprendizaje ubicuo, u-learning (HERNÁNDEZ, 2017; QUICIOS, 2015), subrayándose, al caso, las enormes potencialidades/posibilidades académicas que se desprenden del uso del smartphone en el campo escolar (PEREIRA, GOULART, 2019; HERNÁNDEZ, 2017; RODRÍGUEZ, 2017; MANCINAS, 2018; MEJÍA, 2017; VÁSQUEZ, 2015; BRAZUELO, 2015; MERINO, 2017; PALAZÓN, 2015) a través de las diversas experiencias/iniciativas que se soportan en el aprendizaje electrónico ( $e$ learning) y/o la educación a distancia (EaD). Sirva destacar, de manera especial, que dicho campo enunciativo suele desarrollar, de manera complementaria, un ejercicio de re-significación asociado al papel desempeñado por los sujetos escolares (docentes y estudiantes), en el contexto de esas emergentes condiciones sociotécnicas favorecidas por la irrupción de lo escolar-digital.

El tercer vértice, uno de los más representativos, se estructura a partir del examen de los diversos fenómenos que, asociados a la violencia/acoso, inscriben al smartphone en el marco de esas relaciones de burla, chantaje, agresión y afines, que suelen ser enunciadas bajo la rubrica del cyberbullying. En tales términos, uno de los 


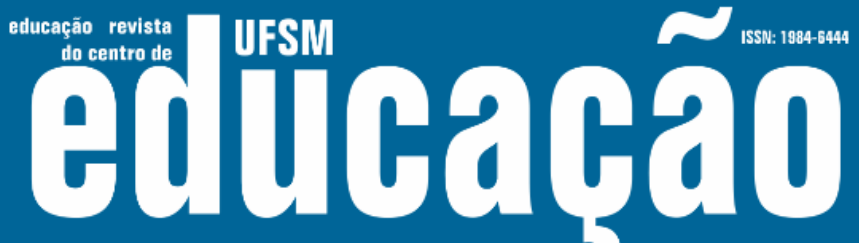

ISSN: 1984-6444 | http://dx.doi.org/10.5902/1984644453236

ejes-enunciativo que figura con claridad al momento de examinar la producción científica sobre el nodo temático objeto de interés, se relaciona con la violencia escolar, con el ciberbullying (LÓPEZ, CORREA, 2019; LUCIO, 2018; DOMÍNGUEZ, 2018, GOMES, 2018; RAMíREZ, 2017), que se materializa a través de las posibilidades de socialización favorecidas, contemporáneamente, por el smartphone.

Al respecto, valga apuntar que tal eje temático se asocia, en virtud de la dimensión político-jurídica inherente al tridente escuela-smartphone-ciberbullying, con el cuarto vértice que aparece, discursivamente, en relación al uso del smartphone, toda vez que el mismo se refiere a las fronteras normativas (GOMES, 2018; DEL CERRO, 2017), que rigen/tensionan, en la actualidad, la polarización que en materia de lo prohibitivo/lo permisivo gestionan el (des)encuentro entre tal dispositivo y lo escolar. En tal sentido, la inclusión o exclusión del smartphone en el campo escolar (en términos legales, institucionales) remite a ese campo de saber, a ese nodo temático, en el cual se fusiona lo político, lo jurídico y lo tecnológico.

En último caso, el quinto vértice identificado se refiere a la dimensión temporal consubstanciada, de manera diáfana, con en el uso del smartphone, toda vez que uno de los temas recurrentes que aparece en los artículos analizados se incardina en esa dualidad que tienen en los usos (tipo de uso) y el tiempo (periodo de uso) como una de sus nodales preocupaciones. De tal manera, el tiempo de uso del smartphone (PEREIRA, GOULART, 2019; CACHEIRO, 2015; GUTIÉRREZ, 2017; QUICIOS, 2015; MERINO, 2017), constituye otro de esos ejes-referenciales fundamentales a partir de los cuales el discurso científico viene enunciando/pensando, de manera particular, lo atinente a la intersección discursiva que resulta de interés.

En tales términos, sea oportuno observar el cuadro número cuatro (Figura 4) titulado Vértices enunciativos, a los fines de visualizar, en términos de los usos escolares del smartphone, cada uno de los apuntados campos de enunciación. 


\section{ussm

ISSN: 1984-6444 | http://dx.doi.org/10.5902/1984644453236

Figura 4. Vértices enunciativos

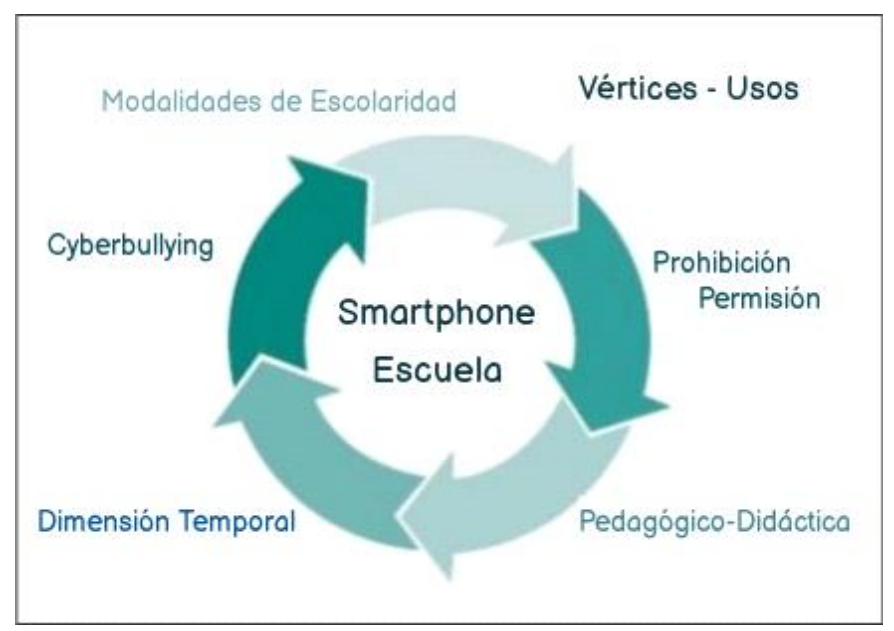

Fuente: Elaborado por los autores

En términos análogos, resulta de interés subrayar las diversas dimensiones, los plurales campos enunciativos, a partir de los cuales se aprecia la criticidad que compone parte de la red semántica, del conjunto de relaciones discursivas, que se han venido puntualizando de manera preliminar, toda vez que tales valoraciones resultan de interés/utilidad a los fines de precisar el campo de dispersión, los particulares sentidos, gracias a los cuales el discurso científico ha venido enunciando/pensando la crítica en lo que compete al eje-temático smartphoneescuela.

De tal suerte, sirva la ocasión para indicar que en cada una de las dimensiones detectadas en lo que se refiere a la crítica de la relación smartphone-escuela, destacaron los siguientes tópicos/señalamientos: en relación a lo patológico (HERNÁNDEZ, 2017; MEJÍA, 2017; DOMINGUEZ, 2018; MERINO, 2017; LUCIO, 2018), se pudo apreciar que el asunto de la adicción, de la dependencia, e incluso de la depresión, constituyen los principales focos de atención, las principales temáticas, que configura la valoración del smartphone desde el punto de vista de las fronteras enunciativas a partir de las cuales se trazan las diferencias entre el uso sano (provechoso, productivo, conveniente) y el uso in-sano (enfermizo, perjudicial, inconveniente) del dispositivo. De igual modo, en sintonía con el cuadro de afecciones aludidas en lo que compete a la patologización del smartphone, otra de las 


\section{Aillbapẫ}

ISSN: 1984-6444 | http://dx.doi.org/10.5902/1984644453236

\section{Consideraciones finales}

En virtud de las regularidades temática, enunciativas, que en el corpus examinado se han destacado de manera preliminar, sirvan los siguientes párrafos para demarcar/puntualizar una batería de consideraciones que se desprenden de la revisión documental, de los análisis, que se han desplegado en la corriente ocasión. De tal manera, valga indicar que:

- El cambio epocal que ha definido el decurso de la última modernidad (de la modernidad tardía y/o líquida), se ha posibilitado, de manera protagónica, por el fraguado de un conjunto de emergentes realidades que tienen en las TIC (de un modo general) y el smartphone (de modo específico), dos de los pilares que sostienen las mudanzas civilizatorias que vienen ocurriendo, de manera tan rápida como impresionante, en cada una de las dimensiones del quehacer contemporáneo. En tal sentido, la escuela del siglo XXI se ha venido redefiniendo a la luz de las diversas dinámicas que, gracias a la expansión de lo digital (sobremanera del smartphone), ocupan un papel cada vez más importante, central, en la emergencia de las nuevas experiencias, de todas aquellas iniciativas que han venido a re-diseñar lo escolar.

-De manera puntual, el discurso científico-escolar producido en el marco del nodo smartphone-escuela gravita, neurálgicamente, en relación al referente usos y la familia de palabras que guardan relación con él. De igual manera, la red semántica que se extrajo de la base documental consultada, permite apreciar que tales usos se encuentran relacionados a cinco vértices enunciativos que corresponden a: el valor pedagógico-didáctico del smartphone; las modalidades de escolaridad de las que participa tal dispositivo, como e-learning, m-learning, y/o u-learning; los fenómenos socio-educativos a los que se le asocia, destacando, de manera central aunque no exclusiva, el ciberbullying y/o la violencia escolar; la dialéctica prohibición/permisión que le define en materia jurídico-escolar; y por último, la dimensión usos/tiempos que tributa para dimensionar la importancia, la penetración del apuntado dispositivo, en la dinámica/cotidianidad de los sujetos escolares. 


\section{Dism

ISSN: 1984-6444 | http://dx.doi.org/10.5902/1984644453236

-Al respecto, bien se puede asentir que el campo de saber definido por la relación smartphone-escuela se despliega en unas condiciones de enunciación, de reflexión, que privilegian el valor instrumental, utilitario, de tal dispositivo, favoreciendo en la escuela (la institución) y los sujetos escolares (docentes, investigadores y estudiantes), unas regularidades discursivas, unas fronteras de enunciación, en las cuales el asunto del poder, la historicidad del smartphone (las relaciones políticas, económicas, sociales a partir de las cuales se produce la existencia de tal objeto), se encuentran desatendidas, invisibilizadas del discurso científico-escolar, ante la hegemonía representada por el valor de uso de la indicada tecnología. La revisión de la crítica escolar que se produce en relación al smartphone confirma, a su vez, el carácter instrumental-utilitario que se ha destacado, de manera preliminar, a lo largo del artículo, toda vez que cada una de las siete (07) dimensiones identificadas en lo atinente al nodo smartphone-escuela (patológica, formativa, disruptiva, institucional, perceptiva, integrativa y procedimental) apuntan, de manera cardinal, al factor-uso como el enunciado central, protagónico, asociado al dispositivo en cuestión. Lo propio ocurre, en tales términos, en lo atinente a los referentes que componen la valoración positiva del smartphone en el campo escolar.

-A la luz de lo suscrito, ciencia y escuela operan como dos instituciones que, al constituirse por diversas y tensionales relaciones de saber-poder, por unas singulares condiciones históricas (agónicas, antagónicas), que resultan sintéticas del tipo de sociedad que procura civilizatoriamente imponerse en el contexto de la modernidad tardía, favorecen una apreciación del smartphone, de lo tecnológico, circunscrita a un topoi que al privilegiar la perspectiva instrumental-utilitaria del dispositivo en cuestión, favorecen una deshistorización del smartphone en virtud de la cual quedan excluidas, invisibilizadas del discurso científico-escolar, las diversas relaciones de poder que convergen, socialmente, en su objetivación. 


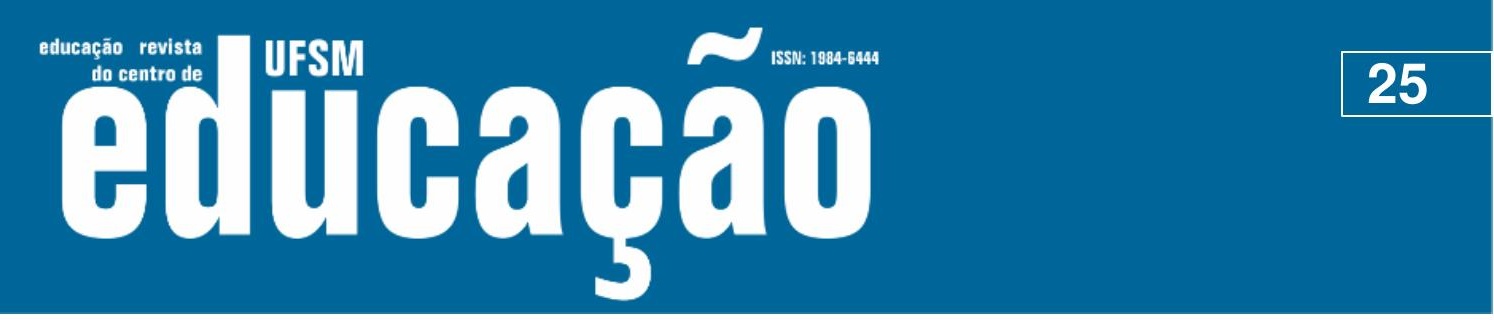

ISSN: 1984-6444 | http://dx.doi.org/10.5902/1984644453236

-De cara a lo apuntado en el párrafo anterior, resulta estratégico apreciar como el discurso científico-escolar imposibilita, a partir de la deshistorización del smartphone, la comprensión de los diversos fenómenos tensionales que a nivel sociológico, económico, político, epistémico, están asociados tanto a la objetivación del smartphone (en términos particulares), como al fraguado de ese tipo de sociedad que se viene imponiendo, civilizatoriamente, al amparo de lo digital.

En tales términos, bien se podría afirmar que el orden discursivo producido en el marco de la relación smartphone-escuela se edifica, en clave nodal, a partir de unas valoraciones, de unos tópicos, de unas regularidades discursivas, que si bien dejan apreciar, por un lado, un campo enunciativo que posibilita la enunciación/intelección del smartphone desde el punto de vista instrumental-utilitario, por el otro desatienden las condiciones de exclusión, dominación y explotación, que concurren en el fraguado del tipo de sociedad que se viene imponiendo, contemporáneamente, en el contexto del capitalismo tardío. He allí la emergencia de un discurso, de unas relaciones de saber-poder, que configuran un sujeto de saber, una institucionalidad, un régimen de verdad, cuya economía de lo decible/pensable juega a favor del tipo de sociedad, del modo de producción, que corresponde a los hegemónicos intereses de la sociedad digital.

\section{Referencias}

ANGENOT, Marc. El discurso social. Los límites históricos de lo pensable y lo decible. Barcelona: Siglo XXI editores, 2010.

BERNSTEIN, Basil. A estruturação do discurso pedagógico. Petrópolis, 1996.

BRAZUELO, Francisco; CACHEIRO, María. Estudio de adaptabilidad para dispositivos móviles en plataformas MOOC. RED. Revista de Educación a Distancia. Murcia, n. 47, p. 01-13, oct. 2015. Disponible en: https://www.redalyc.org/articulo.oa?id=54742306001. Acceso: 11 jun. 2019.

CACHEIRO, María; GARCíA, Francisco; MORENO, Antonio. Las TIC en los programas de Formación Profesional Básica en Ceuta. Revista Apertura. Guadalajara, v. $07, \quad$ n. $02, \quad$ p. $01-18$, oct. 2015. Disponible en: https://www.redalyc.org/articulo.oa?id=68842702011. Acceso: 12 de jun. 2019. 


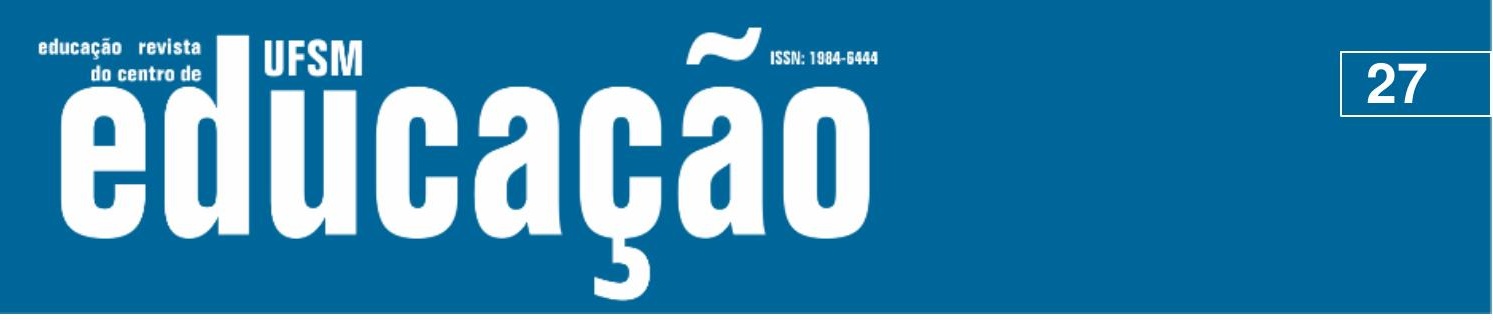

ISSN: 1984-6444 | http://dx.doi.org/10.5902/1984644453236

GUTIÉRREZ, María; SANTANA, Josefina; PÉREZ, Martha. Smartphone: usos y gratificaciones de los jóvenes en México en 2015. Revista Palabra Clave. Bogotá, v. 20, n. 01, p. 47-68, mar. 2017. Disponible en: https://www.redalyc.org/articulo.oa?id=64949843003. Acceso: 13 de jun. 2019.

HABERMAS, Jürgen. Teoría de la acción comunicativa. Madrid: Tecnos, 1987.

HÉRNANDEZ, Nayesia. Uso del teléfono inteligente para el aprendizaje ubicuo en la enseñanza del inglés en una modalidad de educación superior a distancia. Revista de Pedagogía. Caracas, v. 38, n. 102, p. 144-163. 2017. Disponible en: https://www.redalyc.org/articulo.oa?id=65952814008. Acceso: 10 de jun. 2019.

HERRERA, Daniela Ibarra. Topoi como recurso argumentativo en comentarios de blogs de tema político en ciberperiódicos chilenos. RALED. Internacional, v.18, p. 6076. 2018.2 Disponible en: https://raled.comunidadaled.org/index.php/raled/article/view/303. Acceso: 10 de jun. 2019.

HOBSBAWM, Eric. Historia del siglo XX. Buenos Aires: GRIJALBO MONDADORI., 1999.

LÓPEZ, Juan Carlos; CORREA, Marisol Rodríguez. Ciberacoso escolar: experiencias y propuestas de jóvenes universitarios. Revista Iberoamericana de Educación a Distancia. España, v. 22, n. 2, 2019. Disponible en: https://www.redalyc.org/jatsRepo/3314/331460297010/html/index.html. Acceso: 11 de jun. 2019.

LUCIO, Luis; PRIETO, Ma Teresa; CARRILLO, José. Manifestaciones de violencia entre alumnos de educación superior: los usos del WhatsApp. Alteridad: Revista de educación. Quito, v. 13, n. 02, p. 24-37, jul. 2018. Disponible en: http://www.redalyc.org/jatsRepo/4677/467755915004/index.html. Acceso: 11 de jun. 2019.

MANCINAS, Abelardo. Modalidades de cognición en un curso universitario basado en el aprendizaje móvil. APERTURA. Guadalajara, v. 10, n. 01, p. 40-55. 2018. Disponible en: http://www.redalyc.org/jatsRepo/688/68855405003/index.html. Acceso: 10 de jun. 2019.

MARTÍNEZ, José. Obtención del valor de la aceleración de la gravedad en el laboratorio de física. Experiencia comparativa del sensor de un teléfono celular inteligente y el péndulo simple. Revista Eureka sobre Enseñanza y Divulgación de las Ciencias. 2015. Madrid, v. 12, n. 02, p. 341-346. 2015. Disponible en: https://www.redalyc.org/articulo.oa?id=92038753008. Acceso: 12 de jun. 2019. 


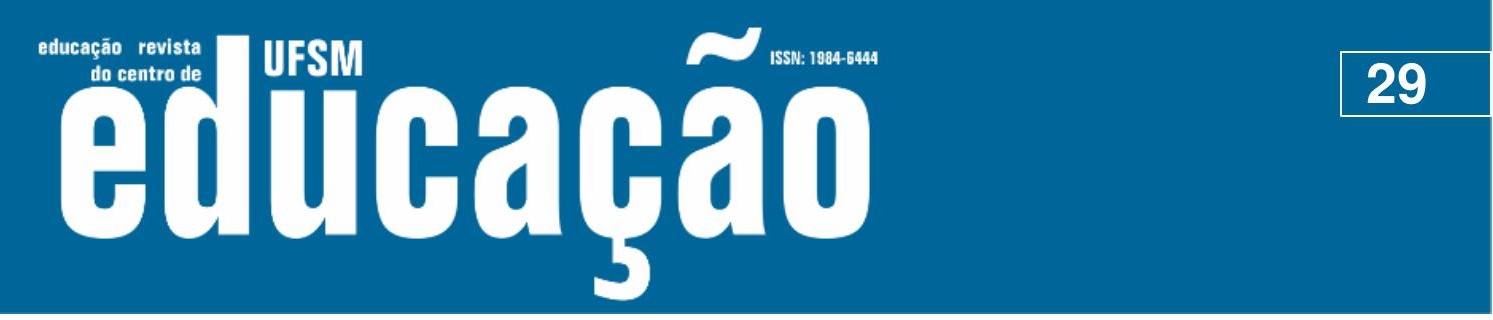

ISSN: 1984-6444 | http://dx.doi.org/10.5902/1984644453236

RODRÍGUEZ, Holmes; RESTREPO, Luis; GARCÍA, Gustavo. Habilidades digitales y uso de teléfonos inteligentes (smartphones) en el aprendizaje en la educación superior. Revista Virtual Universidad Católica del Norte. Medellin, n. 50, p. 126142, may. 2017. Disponible en: https://www.redalyc.org/articulo.oa?id=194250865008. Acceso: 10 de jun. 2019.

RORTY, Richard. El giro lingüístico. Barcelona: Ediciones Paidós, 1990.

SALCINES, Irina; GONZÁLEZ, Natalia. Los smartphones en educación superior. Diseño y validación de dos instrumentos de recogida de información sobre la visión del alumnado. Revista Española de Orientación y Psicopedagogía. Madrid, n. 03, p. 96-120, dic. 2015. Disponible en: https://www.redalyc.org/articulo.oa?id=338245392006. Acceso: 10 de jun. 2019.

TANGARIFE, Diego. La enseñanza de las matemáticas a personas con síndrome de Down utilizando dispositivos móviles. REDIE, Revista Electrónica de Investigación. Baja California, n. 04, p. 144-153, dic. 2018. Disponible en: http://www.redalyc.org/jatsRepo/155/15557980011/index.html. Acceso: 12 de jun. 2019.

VAN DIJK, Teun. Discurso, poder y cognición social. Cuadernos, 1994. Disponible en: https://www.passeidireto.com/arquivo/5826624/van-dijk-teun-a-discurso-poder-ycognicion-social/13. Acceso: 10 de ago. de 2019.

VÁSQUEZ, Esteban; CALVO, Elvira. Adolescentes y cibermedios. Una didáctica basada en aplicaciones periodísticas para smartphones. Estudios Pedagógicos. Valdivia, n. 02, p. 255-270. 2015. Disponible en: https://www.redalyc.org/articulo.oaid=173544018015. Acceso: 10 de jun. 2019.

VÁSQUEZ, Esteban; SEVILLANO, María; FOMBONA, Javier. Análisis del uso educativo y social de los dispositivos digitales en el contexto universitario panhispánico. Revista de Investigación Educativa. Murcia, n. 02, p. 453-469. 2016. Disponible en: https://www.redalyc.org/articulo.oa?id=283346043011. Acceso: 10 de jun. 2019.

WITTGENSTEIN, Ludwig. Investigaciones filosóficas. Madrid: Editorial Trotta, 2017.

YVON, Thomas; LACERDA, Gilberto. Interconexões entre a educação e o smartphone: proposta de um framework contextualizador da aprendizagem nômade. Educação Unisinos. São Leopoldo, n. 03, p. 309-318, dic. 2016. Disponible en: https://www.redalyc.org/articulo.oa?id=449648611006. Acceso: 10 de jun. 2019.

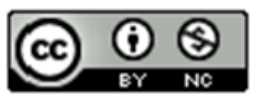

This work is licensed under a Creative Commons Attribution-NonCommercial 4.0 International (CC BY-NC 4.0) 